\title{
Vectorisation intra-oculaire
}

Francine Behar-Cohen

> Les maladies de la rétine sont la première cause de cécité. Les possibilités de traitement sont limitées du fait de la difficulté d'accès à ce tissu, prolongement du système nerveux central tapissant l'intérieur du globe oculaire. Toute stratégie thérapeutique dans le domaine des maladies de la rétine doit comporter une méthode de délivrance de l'agent thérapeutique in situ. Les recherches portant sur les méthodes de délivrance des produits actifs sont de ce fait en plein essor. Elles sont au carrefour de la pharmacologie, de la pharmacotechnie, des biomatériaux, de l'ophtalmologie et de la biologie. Des dispositifs solides, biodégradables ou non, peuvent être implantés dans la cavité vitréenne et des polymères biodégradables peuvent y être injectés. Des méthodes non invasives comme l'iontophorèse ou le transport trans-scléral sont en cours de développement. Les premières applications de ces procédés ont récemment vu le jour en clinique humaine. L'intensification des recherches dans le domaine du transfert intraoculaire de médicaments indique que de véritables perspectives vont s'ouvrir pour le traitement des maladies rétiniennes. <

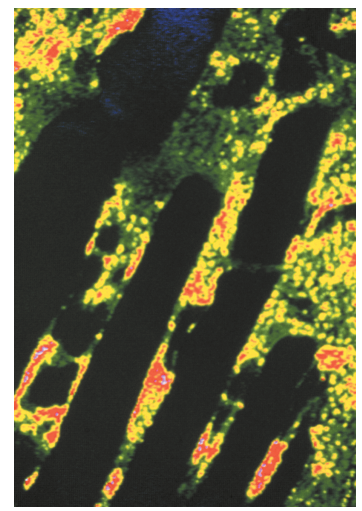

proliférants [6], antiapoptotiques $[7,8]$, anti-inflammatoires ou anti-infectieux), l'avancée thérapeutique est limitée par l'administration et le ciblage du produit actif.

\section{L'œil, un organe isolé de la circulation par des barrières}

La rétine, extension du système nerveux central, tapisse la face interne et postérieure du globe oculaire. Les échanges entre la rétine et la circulation générale se font à travers deux barrières: la barrière hématorétinienne interne, composée de capillaires rétiniens non fenêtrés; la barrière hématorétinienne externe, composée de l'épithélium pigmenté à jonctions serrées. La rétine interne est ainsi vascularisée par un système capillaire hautement sélectif. La rétine externe, contenant les photorécepteurs, est avasculaire, nourrie par la choroïde au travers de l'épithélium pigmenté. Du fait de ces barrières, la biodisponibilité d'un produit administré par voie générale est faible, sauf si le patient est soumis à des perfusions massives (pulse thérapie) ou s'il existe une rupture des barrières hémato-oculaires (inflammation).

D'un point de vue pharmacocinétique, l'œil est considéré comme séparé du reste de l'organisme, le passage systémique d'un produit administré dans l'œil étant quantitativement négligeable. Une modélisation pharmacocinétique propre au segment antérieur est utilisée [9]. Pour le segment postérieur, aucune modélisation pharmacocinétique n'a pu être établie [10].

Voies d'administration utilisées actuellement pour cibler la rétine 
Des injections péri- et intra-oculaires sont utilisées et les premiers dispositifs à libération prolongée ont été évalués. Les injections péri-oculaires (Figure 1) n'évitent pas le passage systémique, seule une faible proportion du produit actif pénétrant directement dans l'œil [11, 12]. Malgré l'absence de consensus, ces injections sont réalisées, parfois avec des formulations non adaptées à l'ophtalmologie. Elles exposent à des complications locales et générales et ne constituent qu'une méthode d'appoint.

L'injection intravitréenne est de plus en plus utilisée. Un produit injecté dans le vitré est éliminé en deux à 18 heures, soit par diffusion antérieure vers l'humeur aqueuse, soit par diffusion transrétinienne vers la choroïde $[9,13,14]$. Des formulations peu solubles peuvent avoir un effet plus prolongé (Kénacort ${ }^{\circledR}$ ). Ces injections doivent cependant être renouvelées pour maintenir un effet thérapeutique, ce qui cumule leurs risques.

Des dispositifs relargants solides, non biodégradables, ont été développés pour éviter la répétition des injections. Ils ont été utilisés pour libérer du ganciclovir chez des patients atteint de rétinite à cytomégalovirus au cours du sida [15] et sont en cours d'évaluation pour la libération de corticostéroïdes [16]. Un dispositif en poly-éthylène-coacétate de vinyle et libérant du ganciclovir (Vitrasert ${ }^{\circledR}$, Baush\&Lomb, États-Unis) a été le seul mis sur le marché. II a pour avantage une libération contrôlée très prolongée. Son principal inconvénient est qu'il doit être implanté et explanté chirurgicalement.

\section{Nouvelles méthodes d'administration en développement}

La recherche s'oriente vers deux voies: le développement de méthodes d'administration locales sans pénétration intra-oculaire, permettant la répétition (iontophorèse ou implantation intrasclérale) et les dispositifs intravitréens biodégradables.

Les systèmes intravitréens à libération prolongée, biodégradables Le système à libération prolongée idéal devrait répondre aux critères suivants: permettre une libération prévisible et reproductible; avoir un profil de libération adaptable à la situation clinique et à son évolution; libérer une concentration homogène de produit actif sans pics indésirables; être facilement implantable et préférablement injectable; être stable, sans déplacement imprévisible dans l'œil; être totalement biodégradable pour éviter l'explantation du système vide; être dénué d'effets toxiques liés à l'exposition prolongée au produit actif, aux composants du système ou à ses produits de dégradation; être facilement manipulable et stérilisable. Aucun système ne répond à tous ces critères. Les avantages et inconvénients des différents systèmes sont brièvement présentés.

\section{Les polymères solides: intérêts et limites}

Les matrices polymériques biodégradables constituées de PLA (polyacide lactique) et PLGA (poly-acide lactique-co-acide glycolique) ont été les plus expérimentés [17-20]. Des implants solides cylindriques PLA/PLGA, injectables sont en cours d'évaluation chez l'homme (0cu- lex, États-Unis). Le risque de ces implants rigides est la survenue de lésions rétiniennes dues à leur mobilité. Les clous scléraux fixés au niveau de la pars plana (partie située en arrière des corps ciliaires à $4 \mathrm{~mm}$ du limbe, en rétine non visuelle et non décollable) présentent donc un intérêt supplémentaire. Une fermeture spontanée de la plaie par un tissu de granulation surviendrait lors de la dégradation du polymère.

Deux mécanismes principaux concourent à la libération d'un produit actif contenu dans une matrice polymérique: l'érosion de surface et la diffusion. Les phénomènes de diffusion sont à l'origine des pics de libération («effet burst») observés avec le PLA/PLGA et dont le contrôle est aléatoire. Pour que le mécanisme d'érosion soit prédominant, le taux de dégradation hydrolytique de surface doit être plus rapide que la vitesse de pénétration de l'eau dans la matrice. De nouveaux polymères ont été conçus dans cette perspective, les polycaprolactones, les poly-anhydrides et les poly(ortho esters).

Les polycaprolactones sont des polyesters dont la dégradation permet une libération du produit actif pendant plus de 6 mois avec une cinétique d'ordre zéro (vitesse de la réaction indépendante de la concentration) [21]. Les poly-anhydrides ont pour avantage une

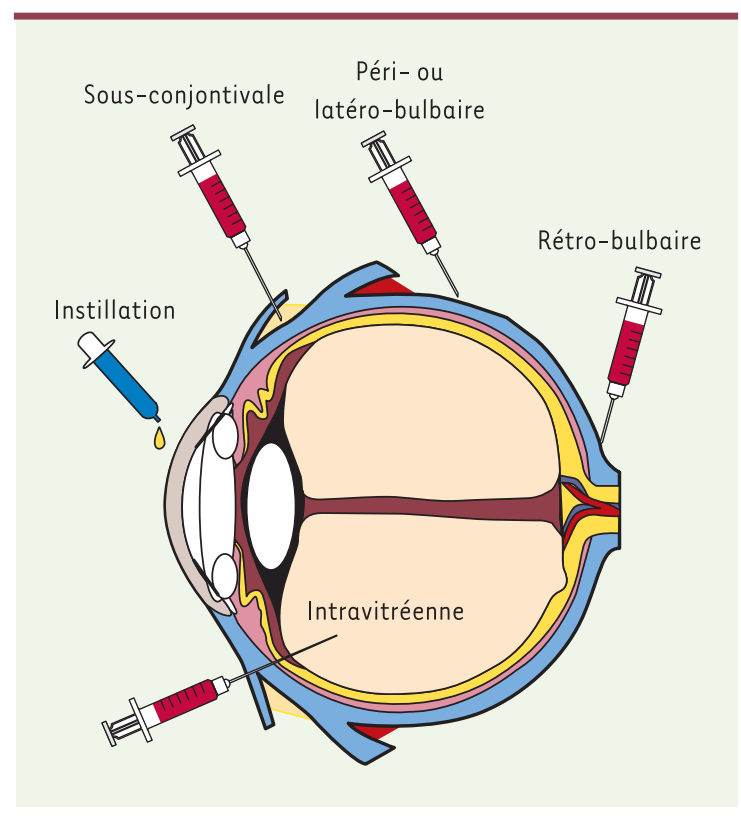

Figure 1. Voies d'administration oculaires des médicaments. Pour traiter les affections touchant le segment antérieur, l'instillation de formulations est habituellement suffisante. Pour traiter les affections touchant le segment postérieur, l'instillation étant inefficace, il est possible d'avoir recours à des injections de produits actifs (corticoïdes ou antibiotiques) autour du globe oculaire, plus ou moins postérieures et plus ou moins proches de la coque sclérale. 
dégradation par érosion sensible au $\mathrm{pH}$ [22].

Ces deux polymères présentent un intérêt par rapport aux PLA/PLGA car ils permettent un contrôle de libération optimisé, mais ils gardent les inconvénients des implants rigides.

\section{L'avenir prometteur des polymères injectables visqueux}

Les poly(orthoesters) (POE) sont une nouvelle famille de polymères semi-solides qui présentent de nombreux avantages pour l'application ophtalmologique, dont une excellente tolérance [23-26]. Leur caractère visqueux permet l'injection directe dans la cavité vitréenne, dans laquelle ils forment une bulle souple et mobile (Figure 2). La libération de produit actif se fait selon une cinétique d'ordre zéro par érosion de surface et le clivage de la liaison ortho-ester étant sensible au pH, la vitesse de dégradation peut être modulée par l'adjonction d'excipients acides ou basiques. Les POE permettent de vectoriser des peptides ou des acides nucléiques ainsi que des composés hydrophobes. La durée de biodégradation complète des polymères varie de huit jours à plusieurs mois en fonction de leur poids moléculaire. Le POE a été évalué pour libérer du 5-fluoro-uracile après chirurgie filtrante (chirurgie de dérivation de l'humeur aqueuse) chez le lapin et en injection intravitréenne et

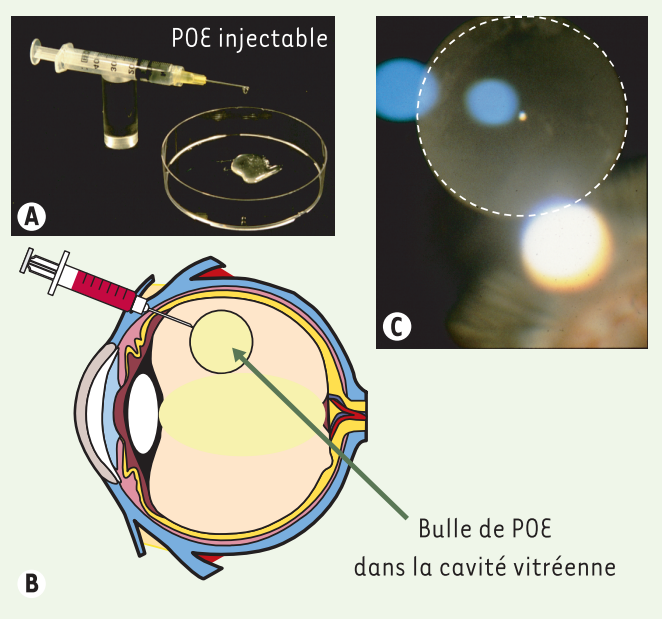

Figure 2. Utilisation d'un polymère injectable dans la cavité vitréenne. Le POE (poly-oethoester), polymère visqueux injectable pour l'application ophtalmologique. $A$. Le polymère se présente sous forme d'un gel translucide injectable ( $\operatorname{Pr}$ Gurny, Université de Genève). B. II permet la libération prolongé d'un produit actif quand il est injecté dans le vitré comme le montre la représentation schématique. $C$. Bulle de POE mobile à la surface de la rétine de lapin (la bulle est entourée d'un trait pointillé). suprachoroïdienne pour cibler les tissus du segment postérieur [27, 28]. Ce type de polymères possède les avantages des polymères dégradables sans les risques des matrices solides et pourrait trouver de nombreuses applications dans le traitement des maladies oculaires.

\section{Particularités et intérêts potentiels des systèmes colloïdaux}

Les systèmes colloïdaux ont l'avantage de pouvoir être injectés avec des aiguilles fines, d'être biodégradables, et d'entraîner une gêne visuelle modérée.

\section{Nanoparticules}

Les nanoparticules ont un diamètre inférieur à $l \mu \mathrm{m}$, en général de 10 à $500 \mathrm{~nm}$. Elles sont constituées de PLA, PLGA, poly- $\varepsilon$-caprolactone, polycyanoacrylate d'alkyle (polycyanoacrylate d'éthyle) ou de polymères hydrophiles comme le chitosan, l'alginate de sodium ou la gélatine. L'intérêt théorique des nanoparticules est leur capacité d'internalisation intracellulaire. Des modifications chimiques de surface permettent d'optimiser leur ciblage cellulaire et de limiter leur élimination par le système réticulo-endothélial. Les nanoparticules sont des vecteurs potentiels de fragments nucléiques et de peptides qu'ils protègent de la dégradation enzymatique. Les nanoparticules ont essentiellement été utilisées pour augmenter la biodisponibilté de produits actifs instillés, dont les résultats étaient mitigés. Peu d'études avaient rapporté l'administration de nanoparticules dans la cavité vitréenne, hormis pour l'encapsulation de ganciclovir dans du polycyanoacrylate d'éthyle. Des travaux récents ont montré qu'après une injection intravitréenne, des nanoparticules (<400 nm) sont éliminées par voie transrétinienne et ciblent préférentiellement les cellules de l'épithélium pigmenté [29, $30]$.

\section{Microparticules}

Injectées dans le vitré, les microparticules (> l $\mu \mathrm{m}$ ) restent en suspension et libèrent le produit actif avec une cinétique contrôlable. Des concentrations efficaces ont été mesurées chez le primate 11 jours après injection [31] et les microparticules ont permis une administration optimisée d'antimétabolites dans des modèles animaux [32].

\section{Liposomes}

Les liposomes permettent de diminuer la toxicité rétinienne de certaines drogues (amphotéricine B) et de favoriser la biodisponibilité de composés hydrophobes (antiviraux, antibiotiques). Le ganciclovir sous forme de liposomes a été utilisé chez des patients sidéens [33]. Des concentrations intravitréennes efficaces ont été mesurés jusqu'à 24 jours, sans aucune toxicité ni complication rétinienne. Les liposomes présentent également un intérêt pour l'apport de fragments géniques, protégés ainsi de l'activité intense des nucléases vitréennes [34]. Après injection, les liposomes s'étalent sur la surface rétinienne et le principe actif diffuse sous forme libre dans les tissus oculaires. Leur élimination se ferait par voie antérieure avec une clairance d'autant plus lente que leur taille est importante [35]. Les liposomes entraînent des opacités vitréennes brillantes responsables de flou visuel et gênant l'examen du fond d'œil pendant plusieurs 
semaines.

\section{Administration locale sans pénétration intra-oculaire}

La voie vitréenne, largement utilisée car facilement accessible, n'est peut-être pas la meilleure pour cibler les structures les plus externes de l'œil et atteindre en particulier la choroïde ou la rétine externe. De nouvelles voies d'administration, fondées sur le concept récemment réactualisé de pénétration trans-sclérale $[36,37]$, visent à obtenir des concentrations élevées dans la choroïde et la rétine et faibles dans le vitré. La sclère couvre $95 \%$ de la surface du globe oculaire $\left(16-17 \mathrm{~cm}^{2}\right)$ et est perméable à des composés hydrosolubles (y compris des protéines). Plusieurs méthodes d'administration tirent parti de la perméabilité sclérale.

\section{L'implantation intrasclérale de biomatériaux}

L'implantation intrasclérale est l'introduction d'un matériau solide ou semi-solide dans les lames collagènes qui forment la coque blanche autour du globe oculaire (Figure 3). Un produit actif déposé à une forte concentration au contact, ou dans le tissu scléral, franchira en partie la barrière hémato-rétinienne externe. Des concentrations biologiquement actives d'anticorps dirigés contre des molécules d'adhérence, administrées à travers la sclère par des mini-pompes osmotiques, ont été mesurées dans la choroïde et la rétine à des concentrations significatives pendant plus de 28 jours, avec des concentrations négligeables dans les milieux oculaires (humeur aqueuse et vitré) [38]. Ces pompes sont difficilement utilisables en clinique. Idéalement, un matériau pouvant être implanté dans la sclère devrait être injectable et prendre une structure gélifiée au contact des tissus comme les gels thermosensibles ou les colles de fibrine. Cette voie d'administration sans risque pour les structures oculaires, pourrait être répétée.

\section{L'iontophorèse trans-sclérale}

L'iontophorèse augmente la pénétration tissulaire d'une molécule, préférentiellement chargée par l'application d'un courant électrique de faible intensité. L'exposition à un champ électrique favorise la mobilité des molécules chargées et agit sur la perméabilité tissulaire et cellulaire. L'idée de l'iontophorèse oculaire n'est pas nouvelle, mais son utilisation chez l'homme est récente. L'iontophorèse transsclérale est efficace pour l'administration intra-oculaire d'agents anti-bactériens, antiviraux [39] et de fragments géniques [40]. Les premiers dispositifs utilisaient des courants non contrôlés, responsables de brûlures [41]. Avec une nouvelle forme de dispositif, respectant les seuils toxiques, des concentrations élevées de corticostéroïdes ont été mesurées dans l'œil [42]. La sonde (à usage unique) est constituée d'un réservoir annulaire posé autour de la cornée, au fond duquel une électrode active permet le passage d'un courant électrique continu de faible intensité (Figure 3). Des premiers résultats cliniques encourageants devront être confirmés par une étude contrôlée [43].

\section{Conclusions}

La multiplicité des systèmes d'administration intra-oculaire évalués reflète la pluralité des situations cliniques et des modalités thérapeutiques possibles. Aucun système ne peut répondre à toutes les exigences, mais le développement de systèmes complémentaires ouvre des perspectives à moyen terme (Tableau I). Le système de délivrance doit être adapté au produit actif choisi, à son mode d'action et ses caractéristiques physico-chimiques, mais aussi à la cible tissulaire ou cellulaire et aux modalités évolutives des processus pathologiques. Le moyen d'administrer des produits actifs pour le traitement des maladies touchant le segment postérieur de l'œil est un défi pour l'ophtalmologie moderne. Relever ce défi impose de poursuivre les recherches visant à renforcer nos connaissances dans le domaine de la pharmacocinétique du segment postérieur. $\diamond$

\section{REMERCIEMENTS}

Je remercie Yves Courtois, Jean-Marie Parel (Bascom Palmer Eye Institute), Robert Gurny (Université de Genève), Jean-Louis Bourges, Riad Bejjani et Mounia Halhal pour leur collaboration dans la réalisation de ces travaux.

\section{SUMMARY}

Drug delivery to target the posterior segment of

the eye

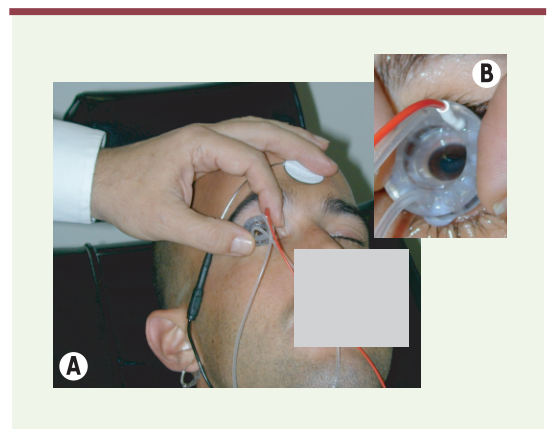

Figure 3. Patient subissant un traitement d'iontophorèse transsclérale. L'iontophorèse transsclérale est une méthode basée sur l'utilisation d'un champ électrique de basse tension qui augmente la perméabilité cellulaire d'une molécule préférentiellement chargée, facilitant ainsi son passage à travers les tissus oculaires jusqu'à la cible. L'idée de l'iontophorèse oculaire n'est pas nouvelle, mais la première utilisation chez l'homme est récente. L'iontophorèse trans-sclérale peut avoir de nombreuses applications pour le traitement des atteintes des segments postérieur et antérieur et s'est avérée efficace pour le transfert de nombreux agents anti-bactériens, antiviraux et de fragments d'acides nucléiques. $A$. l'électrode active est placée sur l'œil, l'électrode de retour est placée sur le front. Le patient est conscient sous anesthésie oculaire locale. B. Sonde d'iontophorèse trans-sclérale (Optis, France) posée à la surface de l'œil chez un patient. 
Retinal diseases are nowadays the most common causes of vision threatening in developed countries. Therapeutic advances in this field are hindered by the difficulty to deliver drugs to the posterior segment of the eye. Due to anatomical barriers, the ocular biodisponibility of systemically administered drugs remains poor, and topical instillation is not adequate to achieve therapeutic concentrations of drugs in the back of the eye. Ocular drug delivery has thus become one of the main challenges of modern ophthalmology. A multidisciplinary research is being conducted worldwide including pharmacology, biomaterials, ophthalmology, pharmaceutics, and biology. New promising fields have been developed such as implantable or injectable slow release intravitreal devices and degradable polymers, dispersed polymeric systems for intraocular drug delivery, and transscleral delivery devices such as iontophoresis, osmotic pumps or intra-scleraly implantable materials. The first clinical applications emerging from this research are now taking place, opening new avenues for the treatment of retinal diseases. $\diamond$

\section{RÉFÉRENCES}

1. Reichel $\varepsilon$, Berrocal A, Ip M, et al. Transpupillary thermotherapy of occult subfoveal choroidal neovascularization in patients with age-related macular degeneration. Ophthalmology 1999; 106: 1908-14.
2. Schmidt-Refurth U, Miller J, Sickenberg M, et al. Photodynamic therapy with verteprofin for choroidal neovascularization cauzed by age-related macular degeneration. Arch Ophthalmol 1999; 117: 1177-87.

3. Hauswirth WW, Lewin AS. Ribozyme uses in retinal gene therapy. Prog Retin Eye Res 2000; 19 : 689-710.

4. Acland GM, Aguire GD, Ray J, et al. Gene therapy restores vision in a canine model of childhood blindness. Nat Genet 2001; 28: 92-5.

5. Liang FQ, Dejneka NS, Cohen DR, et al. Aav-mediated delivery of ciliary neurotrophic factor prolongs photoreceptor survival in the rhodopsin knockout mouse. Mol Ther 2001; 3: 241-8.

6. Ferrara N, Alitalo K. Clinical applications of angiogenic growth factors and their inhibitors. Nature Med 1999; 5: 1359-64.

7. Nir I, Kedzierski W, Chen J, Travis GH. Expression of $\mathrm{Bcl}-2$ protects against photoreceptor degeneration in retinal degeneration slow (rds) mice. J Neurosci 2000; 15: 2150-4.

8. Frasson M, Sahel JA, Fabre M, et al. Retinitis pigmentosa: Rod photoreceptor rescue by a calcium-channel blocker in the rd mouse. Nature Med 1999; 5: 1183-7.

9. Maurice D, Mishima S. Ocular pharmacokinetics in ML. In: Sears ML, ed. Pharmacology of the eye. Berlin: Springer-Verlag, 1984: 19-116.

10. Tojo K, Isowaki A. Pharmacokinetic model for in vivo/in vitro correlation of intravitreal drug delivery. Advanced Drug Delivery Rev 2001; 52: 17-24.

11. Weijtens 0 , van der Sluijs FA, Schoemaker RC, et al. Peribulbar corticosteroids injection: Vitreal and serum concentrations after dexamethasone disodium phosphate injection. Am J Ophthalmol 1997; 123: 358-63.

12. Bodker FS, Ticho BH, Feist RM, Lam TT. Intraocular dexamethasone penetration via subconjunctival or retrobulbar injections in rabbits. Ophthalmic Surg 1993; 24: 453-7.

13. Blair NP, Evans MA, Lesar TS, Zeimer RC. Fluorescein and fluorescein glucuronide pharmacokinetics after intravenous injection. Invest Ophthalmol Vis Sci 1986; 27: 1107-14.

14. Araie M, Maurice DM. The loss of fluorescein, fluorescein glucuronide and fluorescein isothiocyanate dextran from the vitreous by the anterior and retinal pathways. Exp Eye Res $1991 ; 52: 27-39$.

15. Martin DF, Parks DJ, Mellow SD, et al. Treatment of CMV retinitis with an intraocular sustained-release ganciclovir implant: A randomized controlled clinical trial. Arch Ophthalmol 1994; 112: 1531-9.

16. Jaffe G, Ben-Nun J, Guo H, et al. Fluocinolone acetonide sustained drug delivery device to treat severe uveitis. Ophthalmology 2000; 11 : 2024-33.

17. Kimura $\mathrm{H}$, Ogura $\mathrm{Y}$, Hashizoe $\mathrm{M}$, et al. A new vitreal drug delivery system using an implantable biodegradable polymeric device. Invest Ophthalmol Vis Sci 1994; 35: 2815-9.

18. Kunou N, Ogura Y, Yasukawa T, et al. Long term sustained release of GC from biodegradable

\begin{tabular}{|c|c|c|}
\hline & Avantages & Inconvénients \\
\hline $\begin{array}{l}\text { Dispositifs } \\
\text { biodégradables } \\
\text { solides }\end{array}$ & $\begin{array}{l}\text { Ne nécessitent pas de retrait } \\
\text { Absence de modification de la clarté des milieux } \\
\text { Adaptés pour traitements de courte durée (semaines) } \\
\text { Libération contrôlable et prévisible } \\
\text { Implantables, éventuellement injectables }\end{array}$ & \\
\hline $\begin{array}{l}\text { Dispositifs } \\
\text { biodégradables } \\
\text { semi-solides }\end{array}$ & $\begin{array}{l}\text { Injectables } \\
\text { Pas de retrait } \\
\text { Souples, mobiles } \\
\text { Libération contrôlable } \\
\text { Adaptés pour traitements de courte et moyenne durée }\end{array}$ & Possibilité de gène visuelle \\
\hline $\begin{array}{l}\text { Dispositifs } \\
\text { solides non } \\
\text { biodégradables }\end{array}$ & $\begin{array}{l}\text { Pas de produits de dégradation } \\
\text { Pas de modification de la clarté des milieux } \\
\text { Adaptés pour des traitements longs } \\
\text { Libération contrôlée }\end{array}$ & $\begin{array}{l}\text { Nécessité d'implantation et de retrait } \\
\text { chirurgical } \\
\text { Risque d'encapsulation } \\
\text { Corps étranger intraoculaire }\end{array}$ \\
\hline $\begin{array}{l}\text { Nanoparticules, } \\
\text { microsphères }\end{array}$ & $\begin{array}{l}\text { Injectables avec une aiguille } \\
\text { Bon contrôle de libération } \\
\text { En fonction de la taille, possibilité de ciblage cellulaire } \\
\text { Adaptées à la thérapie génique }\end{array}$ & $\begin{array}{l}\text { Effets burst initial et final } \\
\text { Risque d'opacification initiale des milieux }\end{array}$ \\
\hline Liposomes & $\begin{array}{l}\text { Injectables avec une aiguille } \\
\text { Pas de passage dans la rétine }\end{array}$ & $\begin{array}{l}\text { Trouble vitréen } \\
\text { Demi-vie courte } \\
\text { Difficulté de stérilisation } \\
\text { Contrôle de libération difficile }\end{array}$ \\
\hline $\begin{array}{l}\text { lontophorèse } \\
\text { trans-sclérale }\end{array}$ & $\begin{array}{l}\text { Voie externe } \\
\text { Pas de risque pour le patient, ni pour le personnel soignant } \\
\text { Répétition possible }\end{array}$ & $\begin{array}{l}\text { Adaptée pour les molécules chargées } \\
\text { Pas de libération prolongée }\end{array}$ \\
\hline
\end{tabular}

Tableau I. Avantages et inconvénients des nouveaux systèmes de délivrance intra-oculaire de médicaments. 
scleral implant for the treatment of CMV retinitis.

J Control Release 2000; 10:263-71.

19. Hashizoe M, Ogura Y, Takanashi T, et al. Implantable biodegradable polymeric device in the treatment of experimental proliferative vitreoretinopathy. Curr Eye Res $1995 ; 14: 473-7$

20. Kunou N, Ogura Y, Honda Y, et al. Biodegradable scleral implant for controlled intraocular delivery of betamethasone phosphate. Int J Biomat Res 2000; 51: 634-41.

21. Peyman GA, Yang D, Khoobehi B, et al. In vitro evaluation of polymeric matrix and porous biodegradable reservoir devices for slow-release drug delivery. Ophthalmic Surg and lasers 1996; $27: 384-91$.

22. Chasin M, Domb A, Ron $\varepsilon$, et al. Polyanhydrides as drug delivery systems. In: Chasin M, Langer R, eds. Biodegradable polymers as drug delivery systems. New York: Marcel Dekker, 1990: 43-70.

23. Heller J, Barr J, Shen H, et al. Poly(ortho esters)-their development and some recent applications. Eur J Pharm Biopharm 2000; 50: 121-8.

24. Behar-Cohen F, BenEzra D, Einmahl S, Gurny R. Challenge of intraocular dug delivery. Eur J Pharm Rev 2001; 6: 35-40.

25. Einmahl S, Capancioni S, Schwach-Abdellaoui K, et al. Therapeutic applications of viscous and injectable poly(ortho) esters. Adv Drug Deliv Rev 2001; 53: 45-73.

26. Einmahl S, Behar-Cohen F, Tabatabay C, et al. A viscous bioerodible poly (ortho ester) as a new biomaterial for intraocular application. J Biomed Mater Res 2000 , 5: $566-73$.

27. Einmahl S, Behar-Cohen F, D’Hermies F, et al. A new poly(ortho ester)-based drug delivery system for the adjunct treatment of filtering surgery. Invest Ophthalmol Vis Sci 2000; 42: 695-700.

28. Einmahl S, Savoldelli M, D'Hermies F, et al. Evaluation of a new biomaterial in the suprachoroidal space. Invest Ophthalmol Vis Sci 2002; 43: 1533-9.

29. Sakurai $\varepsilon$, Ozeki H, Kunou N, Ogura $Y$. Effect of particle size of polymeric nanospheres on intravitreous kinetics. Ophthalmic Res 2001; 33: 31-6.

30. Bourges JL, Gautier SE, Delie F, et al. Ocular drug delivery targeting the retina and retinal pigment epithelium using polylactides nanoparticles. Invest Ophthalmol Vis Sci $2003 ; 44$ : 3562-9.

31. Peyman G, Convay M, Khoobei B, Soike K. Clearance of of microsphere-entrapped 5 FU and cytosine arabinoside from the vitreous of primate. Int Ophthalmol 1992; 16 : 109-13.

32. Moritera T, Ogura $\mathrm{Y}$, Yoshimura N, et al. Biodegradable microspheres containing adriamycin in the treatment of proliferative vitreo retinopathy. Invest Ophthalmo
Vis Sci $1992 \cdot 33 \cdot 3125-30$

33. Akula SK, Ma PE, Peyman GA, et al. Treatment of cytomegalovirus retinitis with intravitreal injection of liposome encapsulated ganciclovir in a patient with AIDS. Br J Ophthalmol 1994; 78: 677-80.

34. Bochot $A$, Fattal $\varepsilon$, Boutet $V$, et al. Intravitreal delivery of oligonucleotides by sterically stabilized liposomes. Invest Ophthalmol Vis Sci 2002; 43: 253-9.

35. Barza M, Stuart M, Szoka F, Jr. Effect of size and lipid composition on the pharmacokinetics of intravitreal liposomes. Invest Ophthalmol Vis Sci 1987; 28 : 893-900.

36. Ambati J, Canakis CS, Miller JW, et al. Diffusion of high molecular weight compounds through sclera. Invest Ophthalmol Vis Sci 2000; 41: 1181-5.

37. Geroski DH, Edelhauser HF. Transscleral drug delivery for posterior segment disease. Adv Drug Deliv Rev 2001; 52: 37-48.

38. Ambati J, Gragoudas ES, Miller JW, et al. Transscleral delivery of bioactive protein to the choroid and retina. Invest Ophthalmol Vis Sci 2000; 41: 1186-91.

39. Behar-Cohen F, Parel JM, El Aouni A, Chauvaud D. Iontophoresis: The past and the future. J Fr Ophthalmol 2001; 24: 319-27.

40. Voigt M, de Kozak Y, Halhal M, et al. Down regulation of NOSII gene expression by iontophoresis of anti-sense oligonucleotide in endotoxin-induced uveitis. Biochem Biophys Res Comm 2002; 295: 336-41.

41. Lam TT, Fu J, Tso MO. A histopathological study of retinal lesions inflicted by transscleral iontophoresis Graefe's Arch Clin Exp Ophthalmol 1991; 229: 389-94.

42. Behar-Cohen FF, El Aouni A, Gautier S, et al. Transscleral coulomb-controlled iontophoresis of methylprednisolone into the rabbit eye: Influence of duration of treatment, current intensity and drug concentration in ocular tissues and fluids levels. Exp Eye Res 2002; 74: 51-9.

43. Halhal M, Renard G, Courtois $Y$, et al. Iontophoresis from the lab to the bedside. Exp Eye Res $2004 ; 78: 751-7$.
TIRÉS À PART

F. Behar-Cohen 